\title{
Integrated microfluidic tmRNA purification and real-time NASBA device for molecular diagnostics $\dagger+$
}

\author{
Ivan K. Dimov, ${ }^{a b}$ Jose L. Garcia-Cordero, ${ }^{a}$ Justin O'Grady, ${ }^{c}$ Claus R. Poulsen, ${ }^{a}$ Caroline Viguier, ${ }^{a}$ \\ Lorcan Kent, ${ }^{a}$ Paul Daly, ${ }^{a}$ Bryan Lincoln, ${ }^{a}$ Majella Maher, ${ }^{c}$ Richard O'Kennedy, ${ }^{a}$ Terry J. Smith, ${ }^{c}$ \\ Antonio J. Ricco ${ }^{a}$ and Luke P. Lee ${ }^{* a d}$
}

\author{
Received 21st July 2008, Accepted 17th September 2008 \\ First published as an Advance Article on the web 24th October 2008 \\ DOI: $10.1039 / \mathrm{b812515e}$
}

\begin{abstract}
We demonstrate the first integrated microfluidic tmRNA purification and nucleic acid sequence-based amplification (NASBA) device incorporating real-time detection. The real-time amplification and detection step produces pathogen-specific response in $<3$ min from the chip-purified RNA from 100 lysed bacteria. On-chip RNA purification uses a new silica bead immobilization method. On-chip amplification uses custom-designed high-selectivity primers and real-time detection uses molecular beacon fluorescent probe technology; both are integrated on-chip with NASBA. Present in all bacteria, tmRNA (10Sa RNA) includes organism-specific identification sequences, exhibits unusually high stability relative to mRNA, and has high copy number per organism; the latter two factors improve the limit of detection, accelerate time-to-positive response, and suit this approach ideally to the detection of small numbers of bacteria. Device efficacy was demonstrated by integrated on-chip purification, amplification, and real-time detection of $100 \mathrm{E}$. coli bacteria in $100 \mu \mathrm{L}$ of crude lysate in under $30 \mathrm{~min}$ for the entire process.
\end{abstract}

\section{Introduction}

Enzymatic in-vitro amplification of nucleic acids has revolutionised life science research and spurred numerous advances in biotechnology and other disciplines. ${ }^{1-3}$ Nucleic-acid amplification methods and DNA sequence-specific detection now enable precise identification of many pathogens that previously could not be unambiguously determined with traditional laboratory techniques such as culture enrichment, plating, and visual microscopy. Further advantages include precise quantification of target pathogens and real-time detection of amplified products. Nevertheless, conventional detection assays based on nucleic acid amplification provide at best only an order of magnitude lower limits of detection than other conventional methods. ${ }^{4}$ In addition, manual handling and multiple liquid transfers can introduce contamination, which leads to large errors.

A first step toward improving the overall genetic analysis process is to separate, concentrate, and purify target pathogens

${ }^{a}$ Biomedical Diagnostics Institute, National Centre for Sensor Research, Research \& Engineering Building, Dublin City University, Glasnevin, Dublin 9, Ireland

${ }^{b}$ Department of Biomedical Engineering, Universidad de Valparaiso, Chile ${ }^{c}$ Biomedical Diagnostics Institute, National Centre for Biomedical Engineering Science, National University of Ireland, Galway

${ }^{d}$ Biomolecular Nanotechnology Center, Berkeley Sensor \& Actuator Center, Department of Bioengineering, University of California, Berkeley, CA, USA. E-mail: lplee@berkeley.edu

$\dagger$ Part of a special issue on Point-of-care Microfluidic Diagnostics; Guest Editors - Professor Kricka and Professor Sia.

\$ Electronic supplementary information (ESI) available: Silica bead immobilization method; Comparison: Microfluidic RNA purification and real time NASBA protocol versus conventional RNA purification and real time NASBA protocol. See DOI: 10.1039/b812515e from raw samples. Next, the pathogens are lysed and, depending on the sample matrix, volume, and pathogen concentration, an additional step may be necessary to concentrate and purify the nucleic acids. Matrix residues can inhibit amplification, reducing assay efficiency. DNA concentration and purification can enhance the limit of detection of nucleic acid amplification by two orders of magnitude. ${ }^{4}$ Liquid-liquid extraction and solid-phase extraction are two techniques to extract and purify nucleic acids. ${ }^{3}$

Microfluidic lab-on-a-chip technology promises to integrate entire analytical/clinical chemistry processes on monolithic platforms. ${ }^{5-7}$ Compared to conventional laboratory methods, integrated microfluidic platforms offer potential advantages of lower cost, higher speed, smaller sample and reagent volumes, and automation of all processes from sample preparation to analytical result: the "sample-to-answer" concept. ${ }^{8}$ For some bioanalytical measurements, however, the most important consequences of successfully implementing microfluidic lab-on-a-chip technology will be enhanced assay reproducibility and more quantitative results ${ }^{9}$ relative to classical analytical procedures.

Miniaturizing in-vitro amplification of nucleic acids can enable the integration of multiple functional fluidic modules, potentially enabling the analysis of smaller sample volumes, coupled with increased reproducibility, more accurate quantification, and faster analysis. While the number of micro-PCR-related publications has grown rapidly, ${ }^{2}$ many of the reported devices are "stand-alone" structures that replace only the roles of the conventional thermocycler and sample tube. To more fully realise the potential of microfluidic systems, ${ }^{10}$ multiple functions can be combined on one chip. PCR has been integrated in a single microdevice with pre-amplification modules, such as nucleic acid extraction $^{11}$ and purification plus pre-concentration, ${ }^{12}$ or with 
post-amplification analytical modules, such as capillary electrophoresis $(\mathrm{CE})^{13,14}$ and DNA microarrays. ${ }^{15}$ Fully integrated microfluidic PCR devices with pre- and post-amplification modules have also been reported, ${ }^{15-17}$ and portable versions of fully integrated microfluidic PCR devices with all necessary supporting systems, such as pumps and optical detection, have been achieved. ${ }^{18,19}$

Nucleic acid sequence-based amplification (NASBA), a transcription-based RNA amplification system, is more sensitive, rapid, and "user-friendly" than PCR. Initially developed by Compton in $1991,{ }^{20}$ NASBA involves the simultaneous action of three enzymes (avian myeloblastosis virus reverse transcriptase, RNase $\mathrm{H}$, and T7 RNA polymerase) that can produce more than $10^{9}$ copies under isothermal $\left(41^{\circ} \mathrm{C}\right)$ conditions in $90 \mathrm{~min}$. A range of nucleic acid types, including mRNA, rRNA, tmRNA, and ssDNA, as well as nucleic acids from virus particles, can be analysed with NASBA, enabling a range of diagnostics, along with gene expression and cell viability measurements. ${ }^{21}$ In bacteria, tmRNA is universally present and is encoded by the $\operatorname{ssr} A$ gene (10Sa). ${ }^{22}$ It is significantly more stable than mRNA, coming close to DNA in robustness. This high-copy-number target (RiboSEQ) contains conserved and variable sequence regions, making it ideal for application to microbial species identification in molecular diagnostics. ${ }^{23-25}$ The isothermal, low-temperature NASBA process offers multiple advantages relative to the thermal cycling between three temperatures that is required by PCR: it simplifies the thermal design of microfluidic chip, it expands the range of materials and bonding methods that can be used, it increases the number of (thermally sensitive) reagents (e.g., enzymes) or components (e.g., certain valves) that can be included in close proximity on the same chip, and it reduces both the complexity of the instrument and power consumption. A further advantage of NASBA, relative to RT-PCR, is direct amplification of RNA templates without a separate reverse transcription step.

Real-time quantitative NASBA ${ }^{21}$ utilizes molecular beacon probes $^{26-28}$ that specifically hybridize to the target sequence generated during amplification. With suitable optical excitation, the hybridized beacons produce a fluorescent signal proportional to the number of copies of the target sequence produced by the NASBA process, simplifying the analytical procedure and reducing the complexity of the microfluidic chip relative to other detection approaches. The slope of the fluorescence-vs.-time curve can yield a quantitative signal in under 3 minutes with sufficient target copy numbers.

The first successful microfluidic NASBA chip, demonstrated by Gulliksen et $a .^{29}$ (2004) using silicon and glass chambers, included real-time detection. They demonstrated an improvement to the first design in $2005,{ }^{30}$ showing that microfluidic real-time NASBA performed as well as conventional off-chip (in-tube) real-time NASBA.

Key aspects of many sample-to-answer systems include the sample purification, amplification, and detection components. In the case of an RNA analysis system, these elements pose significant technical challenges related to their miniaturisation and integration.

Solid-phase extraction (SPE) is a sample purification technique that can be transferred to microfluidic systems. ${ }^{3}$ Various implementations of SPE in microfluidics have been reported in the literature, but the most efficient microfluidic-based SPE requires complex or in-situ chemistry-based fabrication. ${ }^{3}$ There is a need for simpler fabrication techniques to integrate SPE more easily with microfluidic systems.

To our knowledge, there are no prior reports of integrating RNA purification, NASBA, and real-time detection all on a single chip. Off-chip handling of RNA risks sample degradation, which can compromise assay accuracy, reliability, and reproducibility. To avoid this, the sample preparation, amplification, and detection steps can be integrated in a single microfluidic device. For example, Legendre et al. ${ }^{31}$ showed that a single glass device could extract and PCR amplify DNA from complex lysates of blood, nasal swab, and semen samples; this design was improved by Easley et al. ${ }^{17}$ by adding an electrophoresis channel for PCR amplicon separation and detection. To minimize contamination, different regions of the device were separated by 5 pressure-driven microfluidic valves. With the integration of DNA extraction, PCR amplification, and microchip CE, Easley et al. ${ }^{17}$ demonstrated a genetic analysis system with sample-toanswer capability. However, it is desirable to avoid the complexity of many unnecessary modules such as thermal cycler for PCR or microfluidic CE, and find a solution for effective nucleic acid-based diagnostics.

In this article, we report the monolithic integration of a microfluidic sample-to-answer tmRNA analysis system that includes RNA capture and purification, NASBA, and real-time detection. A novel clog-free bead immobilisation method is used to construct the RNA capture and purification module. The device design also allows for future integration of cell lysis and automated fluid-control functions.

\section{Materials and methods}

\section{Design of integrated microfluidic NASABA chip}

The integrated microfluidic NASBA chip, Fig. 1, is based on microchannels and microchambers, with distinct functional domains: a silica bead-bed RNA purification chamber (RPC, volume $0.25 \mu \mathrm{L}$ ), and a NASBA chamber (NC, volume $2 \mu \mathrm{L}$ ). The remaining channels and chambers have been implemented for the future integration of on-chip chemical lysis. The height of the microchannels and microchambers, $80 \mu \mathrm{m}$, was selected because it is easily achievable with SU-8 2100 photoresist. The RNA purification chamber enables capture of RNA present in the sample by selective adsorption in silica, followed by removal of the remaining crude cell lysate, which includes NASBA inhibitors, by washing.

Detection of amplified tmRNA target from NASBA occurs in real-time via molecular beacons whose fluorescence is unquenched when hybridised to complementary RNA amplicons. The device is characterized using a fluorescence microscope to measure the change in fluorescence from the NASBA chamber as a function of time. The device is mirrored (Fig. 1A) to allow for 2 separate reactions with the same reagents but different samples, nominally to implement control reactions. The relatively large number of inlets (7) and outlets (4) is a consequence of this device being designed as a subcomponent of a larger system we are developing, where a number of the fluidic I/O connections will be replaced with microchannel connections to other components and/or on-chip reagent or waste reservoirs. 

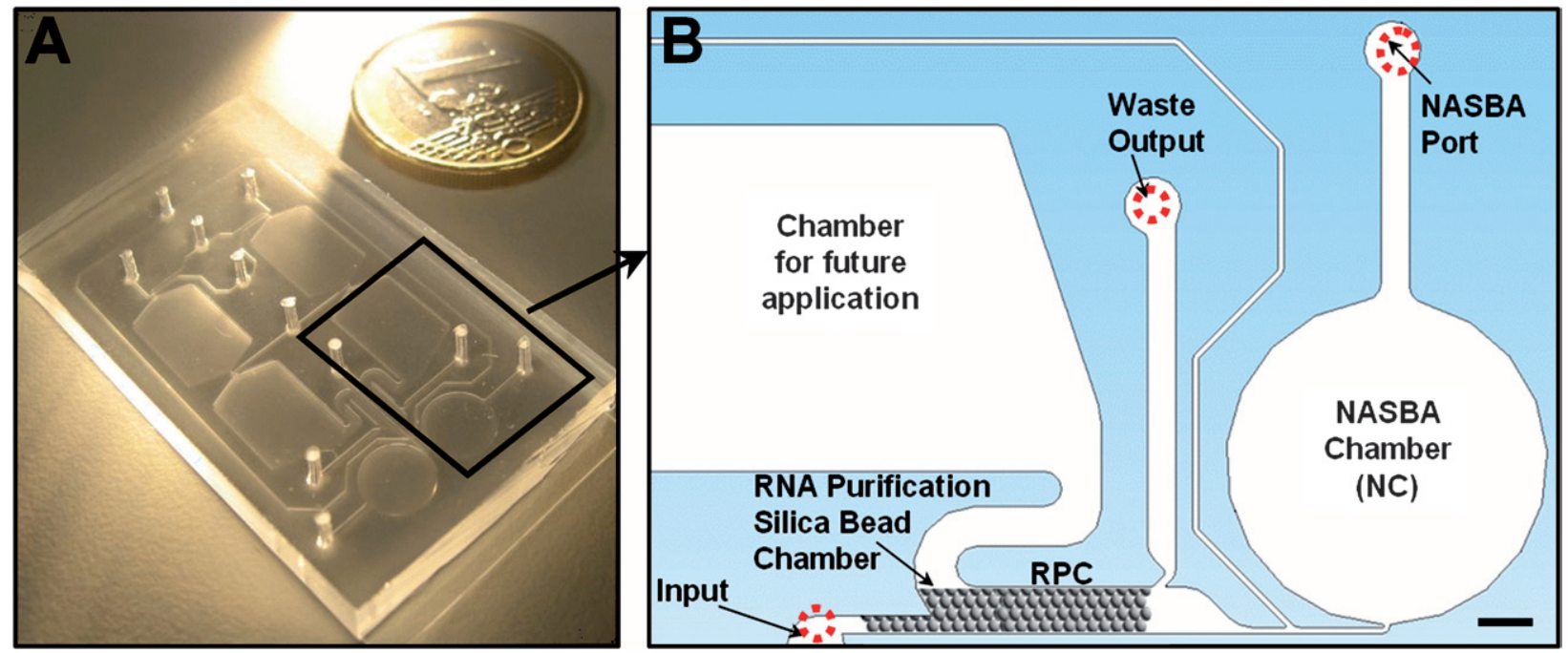

Fig. 1 Integrated microfluidic RNA purification chamber and real-time NASBA device. (A) Photograph of the device. The microfluidic architecture is mirrored to allow for 2 separate reactions with the same reagents, but different samples, to incorporate controls. (B) Single device architecture showing the distinct functional microfluidic modules: RNA purification chamber (RPC) and real-time NASBA chamber. The remaining channels and chambers have been included for future integration of on-chip lysis. All channels and chambers are $80 \mu \mathrm{m}$ high. Scale bar is $1 \mathrm{~mm}$.

\section{Microfabrication}

PDMS is widely utilized for microfluidic devices due to the ease of fabricating relatively complex structures, ${ }^{7}$ its general biocompatibility, and the ease of attaching (sealing) it to glass, silicon wafers, or a second PDMS structure. Microfluidic channels were fabricated using standard soft lithography replica molding techniques. ${ }^{32}$ A mold was created through a single-layer process using negative photoresist, SU8-2100 (Microchem, USA), which was spun onto a clean silicon wafer using a spinner (P6700 Specialty Coating Systems, Inc., USA). The resist $(5 \mathrm{~mL})$ was spread onto the wafer at $500 \mathrm{rpm}$ for $10 \mathrm{~s}$, and the rotation rate was then ramped at an acceleration of $300 \mathrm{rpm} / \mathrm{s}$ to $2,500 \mathrm{rpm}$, at which rate the sample was spun for $30 \mathrm{~s}$ to form an $80 \mu \mathrm{m}$ layer. The wafer was then soft baked at $65^{\circ} \mathrm{C}$ for $5 \mathrm{~min}$ and $95^{\circ} \mathrm{C}$ for $30 \mathrm{~min}$, then UV-exposed for $10 \mathrm{~s}$ at $9.5 \mathrm{~mW} / \mathrm{cm}^{2}$ using a Karl-Süss KSM MJB-55W mask aligner. The wafer was post-exposure baked for $5 \mathrm{~min}$ at $65^{\circ} \mathrm{C}$ and $12 \mathrm{~min}$ at $95{ }^{\circ} \mathrm{C}$, allowed to cool to room temperature, developed in Microposit EC Solvent (Chestech Ltd., UK) developer for $4 \mathrm{~min}$, and finally blown dry with nitrogen. PDMS (Sylgard 184, Dow Corning) was prepared according to the instructions of the manufacturer, degassed in a vacuum chamber for $30 \mathrm{~min}$, then poured on the SU8 mold and cured in a $60{ }^{\circ} \mathrm{C}$ oven for $10 \mathrm{~h}$. The PDMS was then carefully peeled off the mold. Fluid inlets and outlets were punched with a 1 $\mathrm{mm}$ outer diameter flat-tip needle for tube connections. Both a $25 \times 50 \times 0.4 \mathrm{~mm}$ glass cover slide (VWR International Inc., USA) and the PDMS structures were treated with UV ozone (PSD-UV, Novascan Technologies, Inc., Iowa, USA) for 10 min before bonding for $10 \mathrm{~h}$ at $90{ }^{\circ} \mathrm{C}$.

Beads for RNA purification were immobilised within the RPC by loading $3 \mu \mathrm{L}$ of a $68 \%$ (bead: deionised $\mathrm{H}_{2} \mathrm{O}$ volume) solution of $10 \mu \mathrm{m}$ plain silica beads (PSi-10.0, G. Kisker Gbr., Germany) into the input port (IP) (Fig. 2A), with all other ports on the chip sealed except for the waste output (WO). The bead solution was left to dry at room temperature for $3 \mathrm{hr}$, which packs the beads into the extraction chamber. After the beads dried in place, they were immobilised via bonding to the PDMS walls of the

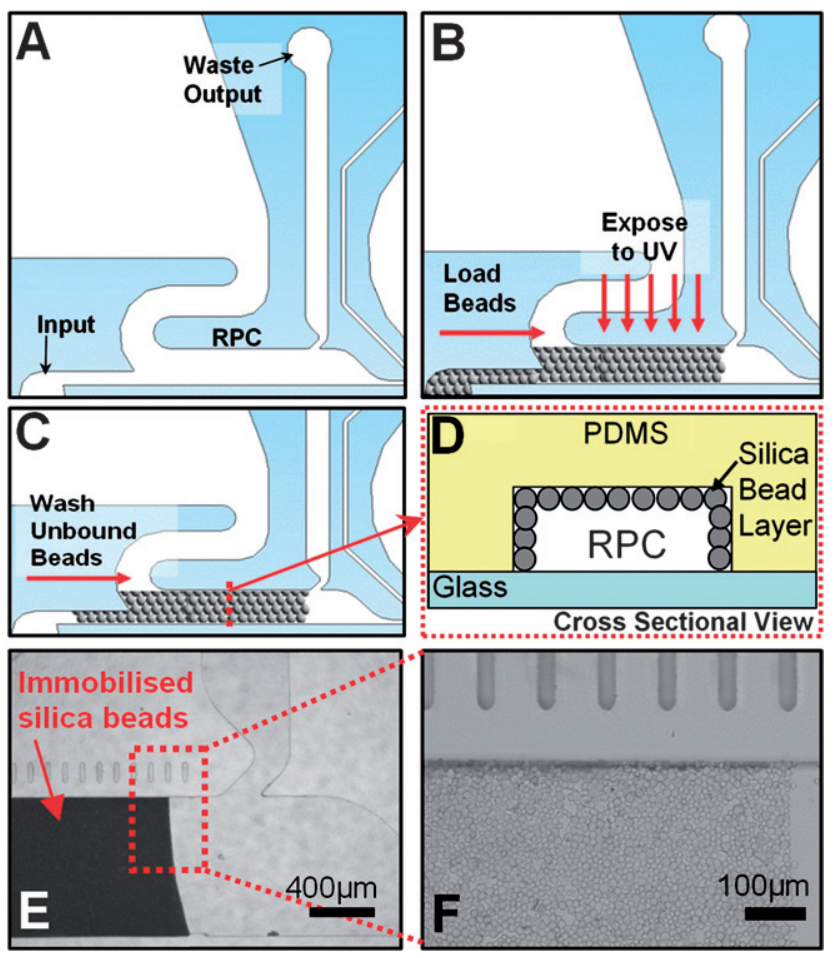

Fig. 2 Method for silica bead immobilisation on PDMS surface. (A) Before loading the beads, all ports are sealed except for the Input and Waste Output. (B) $3 \mu \mathrm{L}$ of plain silica bead solution flows into the input, left to dry, and exposed to UV-ozone for bonding. (C) Unbound beads are washed away with $\mathrm{dH}_{2} \mathrm{O}$, leaving a (D) layer of silica beads bonded to the walls of the RPC (see ESI ). (E-F) Bright-field micrographs of the immobilised $10 \mu \mathrm{m}$ silica beads on the PDMS walls of the RNA purification chamber. 
extraction chamber using UV ozone treatment: the chip was treated with ozone for $10 \mathrm{~min}$ to activate the PDMS, then left to bond overnight at room temperature (Fig. 2B). Finally, a beadwashing step with deionised $\mathrm{H}_{2} \mathrm{O}\left(\mathrm{dH}_{2} \mathrm{O}\right)$ was performed (Fig. 2C) to remove unbound beads and leave a layer of silica beads bonded to the walls of the RPC (see ESI

\section{E.coli culture and lysis}

The E. coli strain XL-1 blue was cultured overnight at $37^{\circ} \mathrm{C}$ with $250 \mathrm{rpm}$ shaking (Gallenkamp orbital shaker, AGB Scientific Ltd., Ireland) in LB medium (Lysogeny broth, also known as Luria broth) containing Tryptone (10 g/L, Lab M Ltd., UK), yeast extract (5 g/L, Lab M Ltd., UK) and NaCl (10 g/L, SigmaAldrich Ireland Ltd., Ireland) made in-house and autoclaved at $121{ }^{\circ} \mathrm{C}$ for $20 \mathrm{~min}$. The cell density was measured as optical density at $600 \mathrm{~nm}\left(\mathrm{OD}_{600}\right)$ on a spectrophotometer (UV-160A, Shimadzu). The number of cells per $\mathrm{mL}$ was calculated from a standard curve based on plate counts.

Cell lysis was performed off chip using MicroLYSIS PLUS buffer (Microzone Ltd., UK) according to the manufacturer's recommendations. An overnight culture of E. coli $\left(\mathrm{OD}_{600}\right.$ of 1.36) with a cell density of $3.4 \times 10^{8}$ colony forming units (CFU)/ $\mathrm{mL}$ (based on plate counts) was centrifuged at 10,000 rpm for 5 min, producing a pellet volume of $5 \mu \mathrm{L}$, and mixed with $40 \mu \mathrm{L}$ of lysis buffer. Temperature cycling was then performed between 65 ${ }^{\circ} \mathrm{C}$ and $96^{\circ} \mathrm{C}$ for a total of $23.5 \mathrm{~min}\left(65^{\circ} \mathrm{C}\right.$ for $15 \mathrm{~min}, 96^{\circ} \mathrm{C}$ for 2 $\min , 65^{\circ} \mathrm{C}$ for $4 \mathrm{~min}, 96^{\circ} \mathrm{C}$ for $1 \mathrm{~min}, 65^{\circ} \mathrm{C}$ for $1 \mathrm{~min}, 96^{\circ} \mathrm{C}$ for $30 \mathrm{~s})$ by transferring tubes between two heating blocks. This crude cell lysate was either used directly for nucleic acid amplification or for RNA purification. For experiments where pure RNA was required for off-chip controls, a commercial RNA purification kit (RNeasy Mini Kit, Qiagen, U.K.) was used according to the manufacturer's recommendations. Cell lysate samples were stored at $-15^{\circ} \mathrm{C}$ until required.

\section{On-chip RNA purification}

The on-chip RNA purification protocol was adapted from previously reported techniques, ${ }^{17,33,34}$ the on-chip protocol is described in the integrated microfluidic device operation section below. To obtain elution profiles, RNA was eluted by flowing $\mathrm{dH}_{2} \mathrm{O}$ through the silica bead chamber and collecting in sequential $5 \mu \mathrm{L}$ fractions. $1 \mu \mathrm{L}$ of each fraction was used for RNA quantification with the Quanti-iT ${ }^{\circledR}$ RNA Assay Kit (Molecular Probes) according to the manufacturer's protocol. The fluorescence was read on an Infinite ${ }^{\mathrm{TM}} 200$ reader (Tecan, Switzerland).

\section{Real-time NASBA}

All reagents required to perform the NASBA were supplied as part of a NucliSens Basic Kit (bioMérieux, UK), except primers and molecular beacon probes, which were supplied by MWG Biotech (Germany). The NASBA mix was prepared according to the recommendations of manufacturer; the final concentration of each primer was $5 \mu \mathrm{M}$, and $5 \mu \mathrm{M}$ for the molecular beacon probe (FAM fluorophore, BHQ1 quencher; detection at $530 \mathrm{~nm}$ ). Both primers and the molecular beacon probe were designed for the specific detection of $E$. coli based on the RiboSEQ platform. ${ }^{35}$
The forward primer was 5'-AATTCTAATACGACTCACTA TAGGGAGATAGTCGCAAACGACGAA- ${ }^{\prime}$, the reverse primer was 5'-CTACATCCTCGGTACTACA-3', and the molecular beacon probe was $5^{\prime}$-FAM-CCAGCTAGCCTGATTAAGTTTTAAGCTGG-BHQ1-3'.

These primers and probes were used for both on-chip and offchip experiments. To allow comparison with on-chip results, offchip real-time NASBA was performed using a LightCycler 480 Real-Time PCR System (Roche Applied Science, USA); the reagent mixture, without enzymes, was mixed with the RNA template and heated on a block heater at $65^{\circ} \mathrm{C}$ for $5 \mathrm{~min}$ and at $41{ }^{\circ} \mathrm{C}$ for $5 \mathrm{~min}$. Next, the enzymes were added, the NASBA mixture was heated for $5 \mathrm{~min}$ at $41{ }^{\circ} \mathrm{C}$, loaded into a 96-well plate, and placed in the LightCycler system. The fluorescence $(530 \mathrm{~nm})$ was measured every minute for $90 \mathrm{~min}$. For each on-chip NASBA experiment, an E. coli tmRNA-positive control and a no-template negative control were amplified off-chip at $41{ }^{\circ} \mathrm{C}$ in the LightCycler in $20 \mu \mathrm{L}$ volumes to verify NASBA reaction mixture performance.

\section{Operation of integrated microfluidic NASBA device}

The RNA purification and NASBA chambers were characterised in this work; therefore, a simple connection scheme was used, in which a syringe pump (Pump 11 Pico Plus, Harvard Apparatus USA) was connected to the I port (Fig. 3A) through a $250 \mu \mathrm{L}$ tube coil (1/32" inside diameter silicone platinumcured tubing, 95802-01, Cole Parmer, USA). The opening and closing functions were accomplished manually using adhesive tape (from Roche LightCycler 480 plates kit, Fannin Healthcare, Ireland) to seal and open ports as required. Prior to use of the device for RNA purification and amplification, the NASBA chamber was filled with $1 \mu \mathrm{g} / \mu \mathrm{L}$ bovine serum albumin (BSA) solution (Sigma Aldrich, USA) and the entire device was soaked in $\mathrm{dH}_{2} \mathrm{O}$ for at least $12 \mathrm{hr}$ at room temperature. Before operation of the device, the BSA solution was rinsed from the NASBA chamber with approximately $1 \mathrm{~mL}$ of $\mathrm{dH}_{2} \mathrm{O}$. The entire device was filled with $\mathrm{dH}_{2} \mathrm{O}$ and all ports except for I and WO were sealed. The I port was connected to the syringe pump with the $250 \mu \mathrm{L}$ tube coil preloaded with the necessary reagents. Between each reagent plug in the tube coil, a $5 \mu \mathrm{L}$ air spacer was injected. ${ }^{36}$ The WO port was connected to waste. Before each experiment, the purification chamber silica beads were conditioned for RNA capture by flowing $100 \mu \mathrm{L}$ of $6 \mathrm{M} \mathrm{GuHCl}$ (Sigma Aldrich) for $20 \mathrm{~min}$ at $5 \mu \mathrm{L} / \mathrm{min}$. Pre-mixed sample solution $(10-40 \mu \mathrm{L}$ of the RNA-containing sample mixed with $90 \mu \mathrm{L}$ of $6 \mathrm{M} \mathrm{GuHCl}$ ) was then pumped through the extraction chamber (Fig. 3A) at $5 \mu \mathrm{L} / \mathrm{min}$. After the RNA bound to the beads (20 $\mathrm{min})$, other substances (cellular debris, proteins, etc.) were washed away by flowing through $100 \mu \mathrm{L}$ of $80 \%$ isopropanol alcohol (IPA) at $17 \mu \mathrm{L} / \mathrm{min}$ (Fig. 3B). After washing left-over IPA is removed and dried with the $5 \mu \mathrm{L}$ air spacer. Next, the NP port was opened and the WO port sealed so that the bead-bound RNA sample could be eluted from the beads by pumping through approximately $11 \mu \mathrm{L}$ of the NASBA reagent mixture (Fig. 3C). All ports were sealed and the mixture was heated to $65{ }^{\circ} \mathrm{C}$ for $5 \mathrm{~min}$ (Fig. 3D) on a peltier device heating system (PE120, Linkam Scientific Instruments Ltd. UK) to denature secondary and tertiary RNA structures that could 

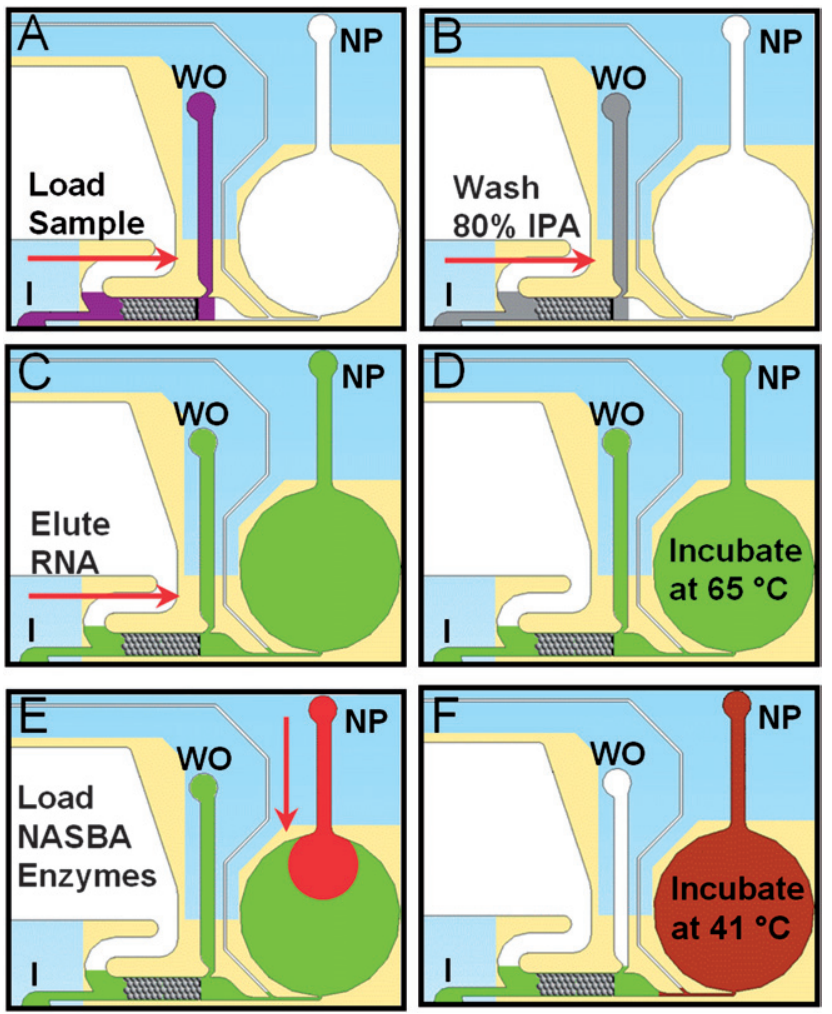

Fig. 3 Representation of integrated microfluidic NASBA operation; all ports except for I, WO, and NP are sealed. (A) Sample mixture is pumped through the RNA purification chamber. (B) Washing solution is pumped through the purification chamber to remove unbound substances. (C) Bound RNA is eluted with primer-containing solution. (D) The mixture is heated to $65^{\circ} \mathrm{C}$ for $5 \mathrm{~min}$. (E) NASBA enzymes are added through the NP port. (F) The fluids are mixed and incubated at $41{ }^{\circ} \mathrm{C}$ to drive the amplification process.

hinder subsequent annealing of primers during NASBA. By opening the I and NP ports and connecting the previously described syringe pump, $2 \mu \mathrm{L}$ of NASBA enzyme mixture were slowly added to the NASBA chamber through the NP port as shown in Fig. 3E. The enzymes were mixed with the reagent mixture by manually applying $40 \times 2 \mathrm{~Hz}$ pressure pulses on the surface of the PDMS over the centre of the NASBA chamber (Fig. 3F).

Next, all ports on the chip were sealed and the chip was mounted on an inverted fluorescence microscope (Olympus IX81) fitted with an incubation chamber (Solent Scientific, UK) and incubated at $41^{\circ} \mathrm{C}$ for $90 \mathrm{~min}$ (a heated microscope stage can serve the same purpose as the incubator). During the incubation time, the NASBA chamber was irradiated once per minute with a $492 \mathrm{~nm}$ excitation beam (excitation filter BP492/18 with a xenon light source, CellR MT20, Olympus) for $230 \mathrm{~ms} ; 530 \mathrm{~nm}$ fluorescent light was sampled through a filter cube (U-MF2, Olympus) with a CCD sensor (Hamamatsu C4742-80-12AG). As the microscope field of view with a $4 \times$ objective covers about $1 / 5$ of the NASBA chamber area, 5 images of the chamber (centre, top, bottom, left, and right side) were obtained for each minute of incubation. Real-time NASBA curves were calculated from the average fluorescence as a function of time from the sets of acquired images. To avoid contamination, each reaction was performed in a previously unused device.

\section{Results and discussion}

\section{Characterization of the NASBA chamber}

The NASBA chamber is the central sensing unit in the integrated device. It was characterized initially by extracting and purifying total RNA from $E$. coli off chip, then preparing serial dilutions of the total RNA and loading into multiple devices. The template RNA dilutions were mixed on chip with the NASBA mix (enzymes, primers, molecular beacons, etc.) as described in the "Real-Time NASBA" section, then pumped to the NASBA chamber. All experiments were performed in triplicate and included a negative control where the RNA template solution was replaced with sterile RNA-free water (Molecular Biology Grade Water, Sigma Aldrich, USA).

Figure 4A shows a clear relationship between the rate of fluorescence increase and the input template RNA concentration. Error bars, the standard deviations of the triplicate measurements, show that the results are reproducible (average variation of $\pm 6 \%$ ). Similar results were obtained from positive control experiments performed on the Roche LightCycler 480. The NASBA chamber has an approximate volume of $2 \mu \mathrm{L}$, one tenth of the standard $20 \mu \mathrm{L}$ NASBA reaction volume. The data suggest that the BSA-treated and water-soaked PDMS chambers

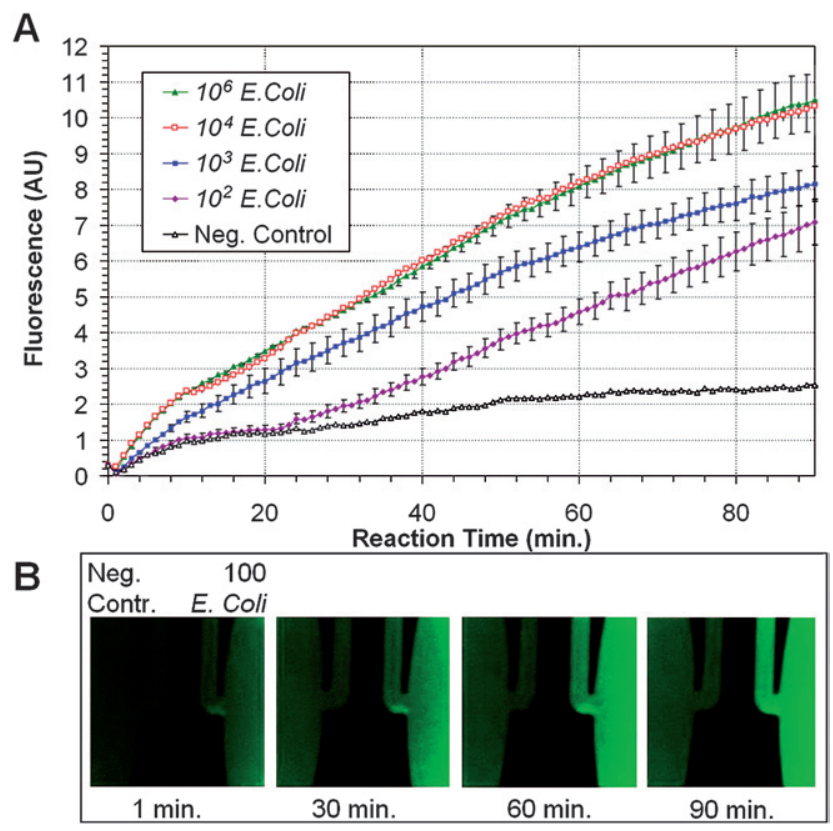

Fig. 4 Characterization of real-time NASBA reaction for off-chippurified E. coli RNA template. (A) Fluorescence intensities in arbitrary units (AU) were plotted over the course of the NASBA reaction. Experiments were performed in triplicate with different numbers of template molecules from total RNA extractions (ranging from $10^{2}$ to $10^{6}$ $E$. coli cells, as indicated). (B) Time-lapse images showing the fluorescence increase of the integrated NASBA chamber entrance loaded with total RNA equivalent to $100 \mathrm{E}$. coli (right of each frame) and the negative control (left of each frame). 
support an efficient real-time NASBA reaction, notwithstanding its greater bio-chemical complexity relative to PCR: NASBA involves 3 enzymes, PCR only one.

\section{Characterization of RNA purification}

RNA loading, washing, and elution procedures were performed as described in the "On-Chip RNA Purification" section. In order to characterize the RNA purification module, RNA was purified from the crude lysate of $10^{7} \mathrm{E}$. coli cells and a total RNA elution profile was obtained by collecting consecutive $5 \mu \mathrm{L}$ aliquots of the eluted solution and measuring the RNA concentration.

While $1 \mu \mathrm{L}$ of each $5 \mu \mathrm{L}$ aliquot was used for RNA quantification with the Quanti-iT® RNA Assay Kit, RNA from another $2.5 \mu \mathrm{L}$ aliquot was amplified by real-time NASBA using the Roche LightCycler 480 and the end-point amplicon fluorescence recorded. The experiments were conducted on two different devices, and are summarized in Fig. 5.

As expected, the results show the amount of RNA is highest in the first elution fraction and decreases as elution continues. The end-point amplicon fluorescence, however, is highest in the $3^{\text {rd }}$ elution fraction (between the 10th and 15 th $\mu \mathrm{L}$ ). The $5 \mu \mathrm{L}$ air spacers that separated the reagents did not cause any problems or bubble formation as they passed through the RPC, because the injected air plugs only passed through straight channels and a soft $90^{\circ}$ turn geometry (from I to WO). Additionally the bead immobilisation method creates a layer of silica beads (Fig. 2D) which leaves plenty of space for debris and air to pass through without leaving bubbles or clogging. Since there is a trade-off between clogging probability and capture efficiency, the height of the purification chamber during manufacture can be adjusted to fine tune the free-flow space to minimise clogging and maximise capture efficiency. Although it was neither required nor used in our application, another advantage of the bead immobilisation

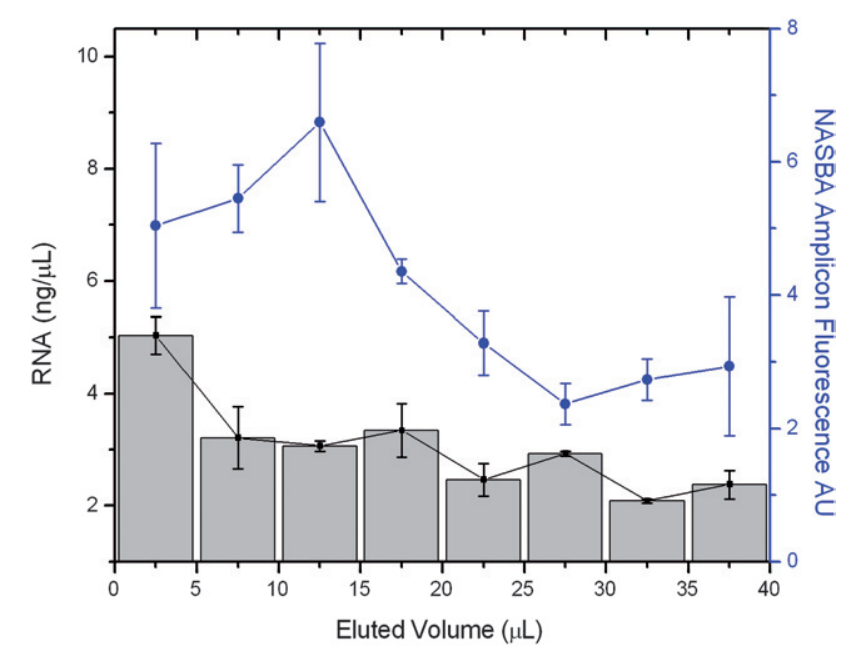

Fig. 5 Elution profile (grey bars with standard error variations) of total RNA extracted from crude lysate of approximately $10^{7} \mathrm{E}$. coli shows total RNA concentration in each $5 \mu \mathrm{L}$ elution fraction. Blue data points with the standard error bars (right $y$-axis) show end-point NASBA amplicon fluorescence after a 90-min off chip real-time NASBA amplification of 2.5 $\mu \mathrm{L}$ of each eluted fraction. method is that it allows the sample solution to flow back and forth over the same beads, increasing capture efficiency; immobilized beads' extraction efficiency can be between $10^{2}-$ to $10^{3}$-fold greater than non-immobilised beads. ${ }^{37}$

Since most inhibitors (including IPA) are washed away, there is a possibility that the elution time for the RNA fragments is size dependent; thus, our target fragments (363-nucleotide tmRNA molecules) mostly elute in the $3^{\text {rd }}$ elution fraction. Similar elution results and differences in amplicon and eluted RNA peaks were reported in a similar microfluidic device. ${ }^{31}$ For the integrated operation of chips, the $3^{\text {rd }}$ eluted fraction was used for downstream on-chip real-time NASBA.

\section{Operation of integrated RNA extraction and real-time NASBA device}

To evaluate the integration of on-chip RNA purification with real-time NASBA, crude lysates of $E$. coli cells (Fig. 6) were prepared. Three $E$. coli lysates from $10^{2}, 10^{5}$, and $10^{8} \mathrm{CFU}$ were prepared together with a negative control $\left(\mathrm{dH}_{2} \mathrm{O}\right)$ as previously described. RNA from the samples was purified and amplified using 4 separate microfluidic chips, one for each sample. Realtime NASBA results, Fig. 6, show successful on-chip integrated RNA purification and real-time NASBA. The time taken for positive samples to reach above background fluorescence during real-time NASBA amplification is known as the time-to-positivity or TTP and can be related to the initial amount of target RNA present in the sample. The lysate from $100 \mathrm{E}$. coli cells was readily detected with a TTP of $<3$ minutes, suggesting a limit of detection significantly below 100 cells. Note that since RiboSEQ targets are used, each E. coli contains approximately 500-1,000 copies $^{23}$ of tmRNA target fragment, so detection of $<100$ cells is not surprising. This high copy number, along with the relatively high stability of tm-RNA, also increases the detection robustness, a critical factor in complex sample matrices such as blood. The difference in end-point fluorescence displayed in Figs. 4A and 6 most likely results from differences caused by the change in the purification capacity between the two experiments; also, the method used to purify RNA is different for the two experiments. These factors may influence the kinetics of the NASBA reaction

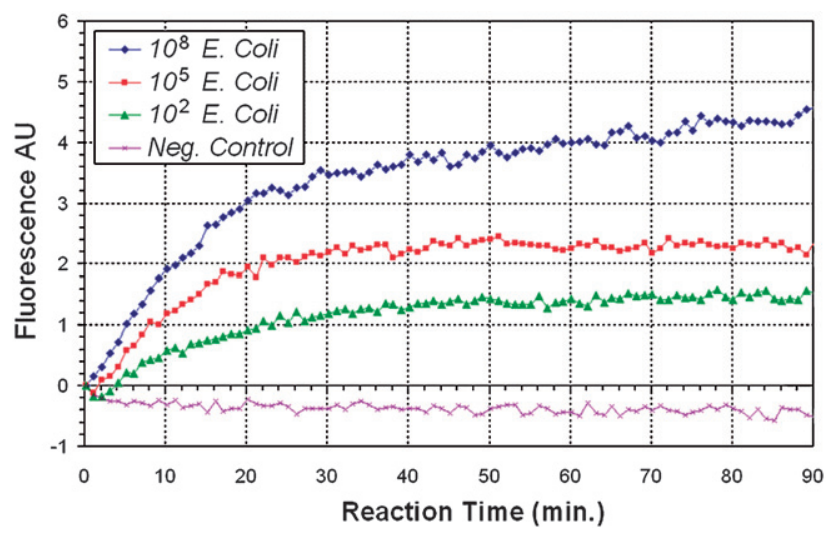

Fig. 6 On-chip RNA purification and real-time NASBA results. Onchip analysis starts by loading crude cell lysate from E. coli, followed by RNA purification, then by NASBA with real-time detection. Each different sample was run on a on a fresh device. 
Table 1 Comparison of on-chip and conventional "off-chip" RNA purification and real-time NASBA

\begin{tabular}{lll}
\hline & \multicolumn{2}{l}{ RNA purification and real-time } \\
& NASBA & \\
\cline { 2 - 3 } & On-chip & Conventional \\
\hline Total reagent volume used & $213 \mu \mathrm{L}$ & $2,450 \mu \mathrm{L}$ \\
NASBA volume & $2 \mu \mathrm{L}$ & $20 \mu \mathrm{L}$ \\
Time to positive & $3 \mathrm{~min}$ & $25 \mathrm{~min}$ \\
\hline
\end{tabular}

and consequently the endpoint fluorescence. Furthermore, the negative control fluorescence in the off-chip purification process (Fig. 4A) is higher (2 AU) than on the on-chip purification (Fig. 6). Since the procedures were similar, the lower negative control fluorescence in the on-chip purification may be due to reduced contamination. In the on-chip procedure, there are no sample pipetting or sample transfer steps after the purification. Fluorophore bleaching can occur during the real-time NASBA reaction; to reduce this effect, the exposure time was limited to $230 \mathrm{~ms}$ for the data shown in Fig. 6.

The TTP for samples of crude E. coli lysate (Fig. 6) demonstrates that the various cell numbers can be distinguished from the negative control in as little as $3 \mathrm{~min}$, which, to the best of our knowledge, is more rapid than any previous report of on-chip amplification and detection from as few as 100 cells. ${ }^{38}$ With this approach, the total time from "sample to answer" can be less than $30 \mathrm{~min}$. Table 1 shows the comparison of on-chip and conventional "off-chip" RNA purification and real-time NASBA. Compared to the conventional off-chip method, onchip NASBA requires approximately 10 times less volume, and the resultant discrimination is approximately 10 times faster ( 3 min on chip, 15-25 min off chip; see ESI ). Relative to conventional, off-chip procedures, on-chip integration reduces the direct pipetting steps from 11 to 2 and the sample-transfer steps from 3 to 1 , reducing significantly the likelihood of sample contamination.

\section{Conclusions}

In this work, we demonstrate the first monolithic microfluidic platform that incorporates tmRNA purification, NASBA-based amplification, and real-time fluorescence detection. A unique new method of silica bead immobilisation simplifies device fabrication, effective sample purification, concentration, and operation. The PDMS-based microfluidic device uses a NASBA reaction volume of $2 \mu \mathrm{L}$, one tenth of conventional NASBA reaction volumes. Result discrimination can be achieved within the first $3 \mathrm{~min}$ after NASBA is initiated, approximately one tenth of the time required in the off-chip NASBA reaction. Direct sample manipulation and pipetting steps are reduced from 11 in the conventional case to 2 in the on-chip case. The fundamental components were characterised and their integrated operation demonstrated with real-time detection of crude $E$. coli bacteria lysates from as few as 100 cells in a time of less than $30 \mathrm{~min}$ from sample loading to answer. The design is not limited to microbial identification applications: it can be adapted for use as a general RNA purification, amplification, and detection platform for point-of-care molecular diagnostics and clinical applications.

\section{References}

1 P. T. Monis and S. Giglio, Infection, Genetics and Evolution, 2006, 6, 2-12.

2 C. Zhang and D. Xing, Nucl. Acids Res., 2007.

3 C. Zhang, J. Xu, W. Ma and W. Zheng, Biotechnology Advances, 2006, 24, 243-284.

4 L. A. Jaykus, Asm News, 2003, 69, 341-347.

5 P. S. Dittrich, K. Tachikawa and A. Manz, Anal. Chem., 2006, 78, 3887-3908.

6 J. Khandurina, T. E. McKnight, S. C. Jacobson, L. C. Waters, R. S. Foote and J. M. Ramsey, Anal. Chem., 2000, 72, 2995-3000.

7 S. K. Sia and G. M. Whitesides, Electrophoresis, 2003, 24, 3563-3576.

8 J. L. Garcia-Cordero and A. J. Ricco, in Encyclopedia of Microfluidics and Nanofluidics, ed. D. Li, Springer, Berlin, Editon edn., 2008, pp. 962-969.

9 S. Nagrath, L. V. Sequist, S. Maheswaran, D. W. Bell, D. Irimia, L. Ulkus, M. R. Smith, E. L. Kwak, S. Digumarthy, A. Muzikansky, P. Ryan, U. J. Balis, R. G. Tompkins, D. A. Haber and M. Toner, Nature, 2007, 450, 1235-1239.

10 L. Chen, A. Manz and P. J. R. Day, Lab on a Chip, 2007, -.

11 C.-Y. Lee, G.-B. Lee, J.-L. Lin, F.-C. Huang and C.-S. Liao, J. Micromech. Microeng., 2005, 15, 1215-1223.

12 J.-G. Lee, K. H. Cheong, N. Huh, S. Kim, J.-W. Choi and C. Ko, Lab on a Chip, 2006, 6, 886-895.

13 N. M. Toriello, C. N. Liu and R. A. Mathies, Anal. Chem., 2006, 78, 7997-8003.

14 C.-S. L. G.-B. L. Fu-Chun Huang, Electrophoresis, 2006, 27, $3297-$ 3305.

15 R. H. Liu, J. Yang, R. Lenigk, J. Bonanno and P. Grodzinski, Anal. Chem., 2004, 76, 1824-1831.

16 L. C. Waters, S. C. Jacobson, N. Kroutchinina, J. Khandurina, R. S. Foote and J. M. Ramsey, Analytical Chemistry, 1998, 70, 158162.

17 C. J. Easley, J. M. Karlinsey, J. M. Bienvenue, L. A. Legendre, M. G. Roper, S. H. Feldman, M. A. Hughes, E. L. Hewlett, T. J. Merkel, J. P. Ferrance and J. P. Landers, PNAS, 2006, 103, 19272-19277.

18 K.-Y. Lien, W.-C. Lee, H.-Y. Lei and G.-B. Lee, Biosensors and Bioelectronics, 2007, 22, 1739-1748.

19 N. C. Cady, S. Stelick, M. V. Kunnavakkam and C. A. Batt, Sensors and Actuators B: Chemical, 2005, 107, 332-341.

20 J. Compton, Nature, 1991, 350(6313), 91-92.

21 G. Leone, H. van Schijndel, B. van Gemen, F. R. Kramer and C. D. Schoen, Nucl. Acids Res., 1998, 26, 2150-2155.

22 K. C. Keiler, L. Shapiro and K. P. Williams, Proceedings of the National Academy of Science, 2000, 97, 7778-7783.

23 B. Glynn, K. Lacey, J. Reilly, T. Barry, T. J. Smith and M. Maher, Research Journal of Biological Sciences, 2007, 2, 564-570.

24 B. Glynn, S. Lahiff, M. Wernecke, T. Barry, T. J. Smith and M. Maher, International Journal of Dairy Technology, 2006, 59, 126-139.

25 J. O' Grady, S. Sedano-Balbás, M. Maher, T. Smith and T. Barry, Food Microbiology, 2008, 25, 75-84.

26 S. Tyagi and F. R. Kramer, Nat Biotech, 1996, 14, 303-308.

27 S. Tyagi, D. P. Bratu and F. R. Kramer, Nat Biotech, 1998, 16, 49-53.

28 S. Tyagi, S. A. E. Marras and F. R. Kramer, Nat Biotech, 2000, 18, 1191-1196.

29 A. Gulliksen, L. Solli, F. Karlsen, H. Rogne, E. Hovig, T. Nordstrom and R. Sirevag, Anal. Chem., 2004, 76, 9-14.

30 A. Gulliksen, L. A. Solli, K. S. Drese, O. Sorensen, F. Karlsen, H. Rogne, E. Hovig and R. Sirevag, Lab on a Chip, 2005, 5, 416-420.

31 L. A. Legendre, J. M. Bienvenue, M. G. Roper, J. P. Ferrance and J. P. Landers, Anal. Chem., 2006, 78, 1444-1451.

32 G. M. W. Younan Xia, Angewandte Chemie International Edition, 1998, 37, 550-575.

33 M. C. Breadmore, K. A. Wolfe, I. G. Arcibal, W. K. Leung, D. Dickson, B. C. Giordano, M. E. Power, J. P. Ferrance, S. H. Feldman, P. M. Norris and J. P. Landers, Anal. Chem., 2003, 75, 1880-1886.

34 B. C. P. Cremonesi, G. Malferrari, I. Biunno, C. Vimercati, P. Moroni, S. Morandi and M. Luzzana, J. Dairy Sci., 2006, 89, $163-169$.

35 M. Maher, B. Glynn, M. Wernecke, J. O'Grady, K. Lacey, S. Lahiff, C. Mullen, S. Sedano, S. McGuinness, T. Smith and T. Barry., in 
Nucleic Acid-Based Technologies, Cambridge Healthtech Institute, Baltimore, Maryland, USA., Editon edn., 2007.

36 V. Linder, S. K. Sia and G. M. Whitesides, Anal. Chem., 2005, 77, 64-71.
37 Y.-C. Chung, M.-S. Jan, Y.-C. Lin, J.-H. Lin, W.-C. Cheng and C.-Y. Fan, Lab on a Chip, 2004, 4, 141-147.

38 L. Chen, A. Manz and P. J. R. Day, Lab on a Chip, 2007, 7, 14131423. 